\title{
EL MODELAJE SOCIAL DEL TIEMPO: LA INSTITUCION ESCOLAR Y LOS ALUMNOS DE ORIGEN EXTRANJERO EN FRANCIA*
}

\section{Ana Vásquez}

El tiempo es tan inasible como el aire que se respira; sin percatamos estamos inmersos en él. Tal vez ello explica por qué el interés por el tiempo apareció tardíamente en las ciencias sociales, salvo algunas excepciones (Hall, 1959; Bachelard, 1963). Pero a partir de los años 70 abundan las investigaciones en las cuales se descubre el modelaje social del tiempo y se pretenden determinar los factores que hoy lo condicionan. Hoy en día sabemos que en cada sociedad el tiempo social se estructura de acuerdo con las necesidades económicas: su organización trata de responder a un proceso de producción más competitivo (Grass, 1979); ello presupone que coexistan al lado de las normas predominantes otras estructuras, según las actividades, clases sociales, regiones geográficas, etc. (Rezsohazy, 1981). Sin embargo, la estructura temporal toma características, histórica y culturalmente determinadas por el grupo social, que le asignan rasgos particulares a su adquisición y organización (Grossin, 1975). Por último, la evolución y las necesidades de las sociedades altamente industrializadas imponen la sujeción del individuo al tiempo; sin importar que en su rígida estructura el tiempo de trabajo sobrepase a los demás tiempos, de diversión, de descanso; sin que importe tampoco que en la actualidad el individuo se enfrente a la paradoja de un tiempo libre tan programado, controlado y rentable como el tiempo de trabajo (Grossin, 1986; Rezsohazy, 1986; Samuel 1984).

Hago este breve recuento de los conocimientos anteriores y de las investigaciones actuales sobre el tiempo, porque mi propio trabajo se inscribe en dicha perspectiva, aunque presenta características específicas en cuanto a la población estudiada y a la metodología empleada.

\section{PERCIBIR LA RELATIVIDAD DE LAS NORMAS TEMPORALES}

Si tomamos como punto de partida la constatación de Hall (op. cit.) en el sentido de que las normas se encuentran incrustadas en la cultura de tal manera que los individuos no son conscientes de ello y las consideran de manera absoluta como las únicas viables, podríamos postular que sólo se pone en evidencia la relatividad social en la estructuración del tiempo cuando individuos o grupos de individuos se ven confrontados a normas sociales diferentes a las suyas.

En efecto, me sorprendieron las anotaciones espontáneas que respecto al "tiempo de los franceses" hicieron unos inmigrantes económicos y políticos de habla hispana y lusitana, que viven en Francia y con quienes adelanté una investigación sobre el proceso de transculturación.

Debo abrir un breve paréntesis con el fin de recordar las características de la transculturación, puesto que un individuo, turista, estudiante, becario, diplomático, etc.

\footnotetext{
"Traducido por Cecilia Pinto de Cáceres a partir del artículo publicado en "LES TEMPS SOCIAUX".

** Investigadora chilena radicada en París.
} 
confrontado a una cultura diferente a la suya, no pasa por el mismo proceso. Podríamos considerar que la transculturación comienza cuando se dan relaciones durables de poder, explícitas o apenas insinuadas, o cuando un grupo se siente obligado a abandonar su cultura para adoptar la del grupo dominante. No se trata simplemente de la adquisición "de una cultura", se trata del abandono simultáneo de las normas y valores que le sirvieron al individuo para socializarse y que tocan a la esencia misma de su yo. Permanentemente, se ve confrontado a la hostilidad de dos sistemas de normas, y al deber de situarse en uno o en otros y continuamente siente que los está traicionando.

Aun cuando se trata de comunidades numéricamente pequeñas, el proceso de transculturación implica siempre la confrontación de dos comunidades. El individuo en proceso de transculturación constantemente debe determinarse respecto de dos grupos de referencia; en nuestro caso los "franceses" y la comunidad de inmigrantes o exilados. Respecto a la segunda, los individuos se definen, adhieren o se separan ya que es el espacio simbólico el que representa la cultura de origen.

¿Será necesario recordar que en todo proceso la transculturación se desarrolla en el tiempo? La percepción de sí mismo y la manera como el grupo y cada individuo en particular "viven" su estadía en Francia se modifica, sin embargo, se pueden diferenciar ciertas etapas (Vásquez, 1983).

En un contexto semejante, la percepción de los dos códigos de normas temporales y la de los valores que se atribuyen son muy agudos como si la organización social del tiempo fuese un obstáculo para cualquier esfuerzo de adaptación a la nueva situación, hecho que a menudo se evidencia por la transculturación, según datos arrojados por esta investigación.

\section{LOS HIJOS DE EXTRANJEROS EN LA INSTITUCION ESCOLAR}

Estudié las modalidades de dicho proceso en la escuela, pues la institución escolar es un mundo de socialización. (Chombart de Lauwe, 1976) fundamental para los niños extranjeros. Efectivamente, ella propone los modelos principales que sirven de referente a la comparación de las normas del país y las francesas.

El enfoque etnográfico (Spindler, 1982; Wilcox, 1982) me parece que se adapta mejor al estudio diacrónico y simultáneo de las normas institucionales y a su ubicación; conviene también al estudio sobre la evolución de las representaciones y comportamientos de actores sociales, personal de la escuela y alumnos todos ellos extranjeros. En efecto, la etnografía facilita las investigaciones durables sobre terreno y permite tomar en cuenta otro tipo de información a menudo olvidada por otros métodos de trabajo como las entrevistas informales y la observación de situaciones pertinentes, no previstas en la investigación; también facilita la estructuración de datos diferentes de acuerdo con criterios que aunque definidos con anterioridad, pueden variar según el énfasis que se haga en una de sus etapas ("escena etnográfica", "incidente crítico", etc.), (Wilcox, op. cit.). La primera encuesta estadística se aplicó en todas las escuelas de un barrio parisino (Vásquez, 1981), a la cual siguieron 70 observaciones sistemáticas, cada una de una duración de media jornada de clase, a lo largo de dos años, en ocho escuelas de París y sus afueras. La observación se centró a) en los docentes como representantes de las normas de la institución, b) en el niño extranjero, español o latinoamericano, 
recientemente radicado en Francia, ocho en total ${ }^{1}, \mathrm{c}$ ) en un niño francés considerado por el docente como "un alumno francés medio" (A.F.M.) y que representa el promedio de la clase $^{2}$; en total 8 niños.

Además, se grabaron unas cien entrevistas semidirigidas con niños lusitano-hablantes e hispano-hablantes, inmigrantes o exilados, niños y niñas cuyas edades oscilaban entre los 10 y los 17 años; todos ellos escolarizados en Francia; solamente una parte había vivido una experiencia previa de escolarización en su país natal. Estas entrevistas se sometieron a un análisis factorial de correspondencias ${ }^{3}$ las opiniones fueron recopiladas y se expresaron en porcentajes que permitieran hacer comparaciones estadísticas ${ }^{4}$. En la segunda etapa se adelantó un estudio, cualitativo y cuantitativo, de la situación de enunciación, con miras a afinar el instrumento de análisis con los aspectos involucrados en el discurso, sobre todo los silencios que podrían llegar a ser bastante reveladores ${ }^{5}$. Numerosas entrevistas informales se llevaron a cabo con los institutores de las clases observadas; de la misma manera se procedió con los directores de dichas escuelas, con el personal de servicio y con otras personas involucradas directamente con esos niños: psicólogos y miembros del G.A.P.P., con institutores de las "clases de iniciación", etc. Dichas entrevistas fueron objeto de notas a posteriori.

De acuerdo con los análisis del tiempo citados arriba, se organizará la presentación de los resultados, primero alrededor del "tiempo de trabajo", la actividad durante la clase; después en torno al "tiempo libre" dentro de la escuela, desplazamientos y sobre todo en el restaurante escolar ${ }^{6}$. Sucede que los niños y adolescentes estudiados en sus representaciones se refieren constantemente a las normas de la cultura de origen; en cada caso se hace necesario resumir brevemente, no las normas en sí mismas sin las representaciones que de ellas se hacen los sujetos.

\subsection{El tiempo de clase}

\subsubsection{Las normas de la institución}

Dos criterios complementarios entre sí, establecen las pausas para el funcionamiento global del sistema escolar francés: la estima del niño se mide de acuerdo con la precocidad con la cual él recorra el currículo escolar; cualquier pérdida de año significa fracaso.

En otras palabras, a un niño mayor o repitente se le orienta "hacia cursos especiales carentes de salida hacia la enseñanza superior"; en este sentido el criterio de velocidad

\footnotetext{
1 Desafortunadamente no fue posible trabajar con niños que hubiesen llegado en la misma época; la variable se ubica en los dos años y algunas semanas (una niñita que acababa de ser escolarizada en Francia).

${ }^{2}$ El criterio de "alumno promedio" es discutible; es posible que esos alumnos representen la idea que el docente se hace "de lo que debería ser un alumno promedio" y no "el promedio real" de la clase. En todo caso, el comportamiento de esos alumnos es bastante homogéneo: se trata de niños relativamente juiciosos desde el punto de vista "disciplina" y que obtienen notas promedio.

${ }^{3}$ Este trabajo se realizó en el Laboratorio de Estadística Matemática de la Universidad de París VI, con 8. Soukup-Stepan. Ver referencias bibliográficas adelante.

${ }^{4}$ Se calcularon $X(2)$ en los subgrupos (sexo, origen, causa de movilización y edad: pequeños medianos y grandes). Cuando X(2) era significativo, se calculaba el coeficiente del grupo (c).

${ }_{6}^{5}$ Dicho trabajo se llevó a cabo con el psicolingüista M. Praux. Ver referencias adelante.

${ }^{6}$ Intentamos observar a esos niños durante el recreo, pero nos fue imposible definir un cuadro que permitiese comprender su comportamiento respecto del tiempo. En ese sentido nuestras observaciones son cualitativas.
} 
lleva implícito el de éxito escolar. Sin embargo, no existen normas explícitas relativas a la organización del tiempo dentro de la clase; para efectos de nuestra investigación, se dedujeron del comportamiento observado en los institutores (No. 16) y de las entrevistas que con ellos sostuvimos.

Los institutores observados emplean métodos bastantes directivos, de tal manera que los alumnos se organizan de acuerdo con el exclusivo criterio del institutor. Cabe anotar los frecuentes ejercicios en los cuales se impone un plazo de realización, breve y controlado. A excepción de uno de los docentes, todos los demás insisten en la velocidad; en las setenta observaciones exigieron velocidad 241 veces; podemos ilustrarlo con ejemplos relativos a la velocidad del trabajo "Rápido", "No les toma más de dos minutos" "Rápido, rápido japúrate!" "Ahora rápido; cada uno solo!".

Paralelamente, la "lentitud" aparece como un defecto. Anotamos algunas observaciones desmotivantes como: “†Tú, como siempre, trabajas despacio!" ". . .No necesitas media hora para voltear la página”. “ ¡Pero claro, el señor es siempre el último!”. En la mayoría de las veces las entrevistas se centraron en los niños extranjeros observados: sólo hubo una excepción; todos los demás se quejaron de su "lentitud" y basándose en ello, manifestaron sus dudas en cuanto a posibilidades de éxito escolar.

\subsubsection{Los niños extranjeros y su representación del tiempo de "Allá"}

Durante las entrevistas los niños recurren al tiempo de "allá" en oposición a las normas francesas, aún en el caso de no haber sido escolarizados en su país natal. Claro está que se pueden hacer numerosas críticas a los países de esos niños; sus comentarios se refieren a una imagen idealizada de su país de origen ${ }^{7}$ y de su escuela.

En cuanto a los niños latinoamericanos, cabe anotar que el período anterior al exilio, en la historia de dichos países, fue un período de expansión cuantitativa, y de una extraordinaria democratización escolar, tanto a nivel administrativo como pedagógico. En el momento en el cual se adelantó nuestra investigación, un fenómeno similar sacudía a los hijos de inmigrantes: el florecimiento de la "revolución de los claveles" en Portugal y las secuelas de la muerte de Franco en España ofrecían nuevas perspectivas en las escuelas. De otro lado, en esos países, el tiempo del cual dispone el niño para concluir sus estudios no es demasiado corto; el repetir un curso puede considerarse como un "tiempo de maduración" y no como un fracaso. En las entrevistas, a partir de ese momento el tiempo de la escuela del país se presenta como un contramodelo: "Allá los maestros preferíamos que nos tomáramos nuestro tiempo para hacer las cosas; se trataba de hacerlo bien. Aquí, lo único que cuenta es hacerlo rápido". "Aquí lo importante es aprender de memoria, sin pensarlo. Allá había que reflexionar y ser capaz de criticar". "Como aquí todo debemos hacerlo rápido, uno sólo piensa en sí mismo; allá se era ante todo solidario; te detenías a ayudar a tu compañero"8.

\footnotetext{
${ }^{7}$ Los hijos de inmigrantes de la península lbérica regresan con regularidad a su país para pasar vacaciones; su imagen se asocia con verano y placer. Al contrario para los exilados, el regreso al país está prohibido; para estos niños y adolescentes, el país representa su pasado, mitificado por los adultos de la comunidad y por ellos mismos; el exilio, o el comienzo de exilio significa una modalidad social regresiva para la mayoría de ellos: el país perdido es sinónimo de felicidad.

${ }^{8}$ Sin embargo, debemos hacer notar que son ante todo los niños de exilados quienes expresan sus críticas a la organización del tiempo de clase en Francia; son quienes idealizan el tiempo de clase "allá". Dichas diferencias no se explican por el medio de origen: los hijos de ejecutivos y de obreros expresan la misma crítica. En general los niños de inmigrantes se cohiben mucho para criticar la organización de la clase; prefieren criticar el tiempo libre" dentro de la escuela.
} 


\subsubsection{Los niños de extranjeros y su percepción de la organización del tiempo en clase}

Numerosos niños se refirieron a la manera como los profesores exigen una mayor velocidad en la realización de las tareas escolares y, (un 76,23\%: ver Vásquez, 1982), una amplia mayoría piensa que los docentes insisten constantemente en la velocidad que, de esta forma, llega a ser una cualidad esencial de lo que sería un buen trabajo escolar'

Los niños, y sobre todo los más grandes, mencionaron igualmente el ritmo acelerado y sus repercusiones en el aprendizaje. Según ellos, el control del tiempo, muy breve, lleva al alumno a adoptar un ritmo de carrera, sin poder revisar o corregir su trabajo, sin poder distraerse, ni discutir con sus compañeros. Insisten en el cansancio que sienten por tener que mantener ese ritmo en clase; experimentan una "sensación de vacío" porque no han tenido "tiempo para preocuparse por los demás".

Esta última anotación compartida con otros, pone en evidencia de qué manera la organización del tiempo rebasa la ejecución de las tareas estrictamente escolares y se extiende al conjunto de la organización social: para estar en condiciones de mantener el ritmo y los plazos requeridos, el alumno no puede distraerse; por fuerza debe aislarse de sus compañeros, no verlos ni escucharlos: debe actuar solo.

También analizamos la enunciación, para poder captar, bajo otro ángulo, la estructura del discurso de esos niños (Vásquez y Proux, op. cit.). Recordemos que esas entrevistas semidirectivas comenzaban con un comentario-pregunta: “. . . En la escuela, ¿cómo vas?". No se hicieron preguntas directas sino que pretendimos seguir el discurso del niño respecto de lo que acontece en el colegio. En este análisis seleccionamos nueve palabras que por su alta frecuencia las consideramos palabras claves. Ahora bien, la que aparece más Rápido y Rapidez, por lo menos tres veces en el discurso de cada sujeto. Ello nos permite comprobar en qué medida la organización del tiempo en la escuela tiene un enorme impacto sobre esos niños, y de qué manera el tiempo aparece asociado a la velocidad. A nuestro modo de ver esta palabra caracteriza, para ellos, la organización del tiempo en Francia: hablar del tiempo, hablar en el tiempo, llega a ser sinónimo de velocidad; "actuar con rapidez" es su definición de la escuela. Escojamos dos frases significativas: "la profesora de ciencias llega el primer día de clases y dice: el programa es una carrera contra reloj", "le pido explicación al profesor y me responde, ¡no tengo tiempo!“.

A esta palabra le sigue en frecuencia de uso estar retrasado o llegar tarde. Podemos pensar que, en primera instancia, caracterizaron el "tiempo francés", para, a continuación, situarse respecto a ese tiempo. Manejar las diferencias que se consideran adecuadas en relación con la hora dada depende de cada cultura; esos niños fueron socializados de manera diferente: en sus países respectivos serían "puntuales" cuando en Francia están "retrasados".

Si la segunda palabra deja trazas de la situación de esos niños, la tercera miedo expresa sus sentimientos frente a los individuos y las instituciones que representan esas normas: en el colegio constantemente temen al insulto; un grupo reducido habla incluso de angustia.

\footnotetext{
${ }^{9}$ No entraré en detalles; existen diferencias de opiniones según la edad, origen, pero ante todo, entre hijos de exilados e hijos de inmigrantes. Por el contrario, prácticamente no existen diferencias en cuanto al sexo. Estos aspectos fueron estudiados detalladamente en Vásquez 1983, y en Vásquez y Proux, 1984. (Ver referencia bibliográfica).
} 


\subsubsection{El comportamiento en clase}

Un esquema de seguimiento (Vásquez, 1980) permitió observar el comportamiento verbal y no verbal de los niños extranjeros y el niño representativo del "Alumno Francés Medio"; pudimos comprobar cuántas veces los unos y los otros llegaban "a tiempo" para seguir las indicaciones de los maestros. Los niños de extranjeros se retrasaron 214 veces mientras que el A.F.M. sólo lo hizo 23 veces.

Un análisis del comportamiento de cada tarea demuestra que los A.F.M. tienden a concentrarse más en el trabajo que los niños de origen extranjero. Estos últimos pierden tiempo prestando útiles escolares: borradores, reglas, etc.: 67 veces contra sólo 7 veces de los A.F.M.; o simplemente discuten con otros niños: 178 veces contra 13 de los A.F.M. Dicho comportamiento es mal visto por los docentes quienes lo interpretan como "desorden", sobre todo porque en la mayoría de los casos esos niños disponen de todos los elementos necesarios para su trabajo: el préstamo es, pues, inútil. Bien es verdad que esos niños pretenden simplemente establecer intercambios verbales con otros alumnos: 178 veces contra 13 para los A.F.M.; ellos responden más bien a un modelo de comportamiento en clase que difiere del modelo usual en Francia, y no al deseo de molestar; efectivamente, las relaciones sociales - conversaciones, saludos, etc.- son más apreciadas en su país que en Francia. Algunos maestros interpretaron esos comportamientos diciendo que se trataba de niños que se cansaban más, pero esta hipótesis es inexacta: comparamos su comportamiento en la primera y en la última hora de la media jornada de clase y no encontramos diferencias significativas. Esto quiere decir que las características del comportamiento de esos niños son constantes. Dicho resultado refuerza la teoría de un modelo de socialización escolar diferente del usual en Francia. De cualquier manera, muy pronto esos alumnos se percatan de que su comportamiento es rechazado por el maestro e interpretan sus injurias como una censura a sus relaciones sociales. Por ello en casos aislados, y con frecuencia, hacen cosas diferentes a aquellas que se les ordenó: leer, dibujar, mirar por la ventana, mirar al compañero de al lado, inventarse juegos, etc.; 475 veces contra 25 para los A.F.M. Refiriéndome a la afirmación de los docentes respecto de la lentitud de esos niños me gustaría anotar que ellos sostienen que son lentos porque "terminan después de los demás"; si fuese verdad no son lentos en la ejecución de la tarea; simplemente se organizan según otros objetivos y modelos.

Antes de concluir ese capítulo me gustaría insistir en cómo es de difícil el cambio en las normas del tiempo que estructuran el aprendizaje, para niños y adolescentes socializados en una cultura diferente. Cabe subrayar además que los docentes no alcanzan a darse cuenta de la índole de los problemas vividos por sus alumnos. A menudo los juzgan mal —lentos, desordenados, charlatanes, de mal carácter, volubles, etc.-, o juzgan mal a sus familias - no se preocupan por ellos, no se interesan en sus asuntos, no les dan pautas de disciplina, etc.-

\subsection{El tiempo libre}

\subsubsection{Las llegadas y salidas}

Las entradas y salidas señalan el paso del tiempo de clase al tiempo libre. En las observaciones se anotaron ${ }^{10}$ cada una de las veces que el niño extranjero y que su

\footnotetext{
${ }^{10}$ Fueron clasificadas en dos categorías: "a tiempo" cuando los individuos entraban y salían con el tiempo de la clase; "retrasados" cuando son los últimos, a quienes el institutor debe esperar. 
homólogo francés (el A.F.M.) a) llegaban a tiempo al colegio, a clase, después del recreo ${ }^{11}$ y b) o salían a tiempo al recreo, al restaurante o para regresar a su casa.

En general los A.F.M. entran y salen con el grupo - -148 veces "a tiempo" contra 53 veces "retrasados"--; en cambio los niños de extranjeros son generalmente los últimos en hacerlo -9 veces "a la hora", 104 veces "retrasados"-. ¿Por qué?.

¿Cómo se las arreglan para llegar siempre tarde. La llegada en la mañana es un ejemplo. Llegan corriendo a la hora del cierre, e incluso después, o se quedan en el patio hasta el final. En los recreos son de aquellos que siguen jugando después del toque de campana, suben de últimos, siempre tienen algo que hacer antes de entrar a clase: buscar alguna cosa en el bolsillo, tomar agua, limpiarse los zapatos, etc. Sin embargo, hubiéramos podido esperar una mayor rapidez en las salidas, pero no se vio ninguna diferencia entre "entradas" y "salidas": Cuando sus compañeros se levantaban para salir siempre estaban haciendo una cosa interesante: - terminar la tarea que les dejó el profesor, leer un libro, jugar, solos o con un compañero, etc.- . Seguían entretenidos como si no se hubiesen dado cuenta de nada. Durante las entrevistas un $52 \%$ decían que "llegaban a veces, a menudo o siempre tarde". Por sus explicaciones se comprende que no perciben la necesidad de llegar a la hora exacta. Por otro lado, una minoría rechaza las normas francesas: "A veces llego tarde a propósito, lo hago por el simple deseo de cuestionar su reglamento". (Niño de 15 años). "A menudo me propuse llegar con una hora de atraso a la escuela. La prefecto de disciplina estaba como loca, pensaba que estaba enferma". (Niña de 14 años).

\subsubsection{El tiempo de la comida}

Un poco más del $30 \%$ de los niños no comen en el restaurante escolar, principalmente los hijos de inmigrantes. Por eso no todos los niños estudiados fueron observados en el comedor. Claro está que la comida allí no es un modelo de comida francesa; desde muy temprano, y para la mayoría de los niños escolarizados en Francia, esa es una experiencia del "comer social", y su funcionamiento llega a ser el modelo que cada uno opondrá a aquel que conoce en su familia.

En primera instancia se presentan las entrevistas; en seguida un resumen de las observaciones hechas. Hablando de las clases cabría anotar que quienes mas critican son los niños de exilados; los de inmigrantes expresan las mayores críticas cuando se trata de los comedores escolares.

Cuando se habla del restaurante todos tienen algo que decir y sus opiniones pueden ser consideradas como críticas. Los "no me gusta", "es desagradable", me dan náuseas" se oyen con frecuencia; la crítica se refiere tanto a la alimentación como a la manera de comer; sólo analizaremos el último aspecto. Por lo menos la mitad se queja abiertamente del tiempo del que disponen para comer. ("Apenas estamos comenzando el plato de entrada, cuando la empleada lo recoge regañándonos porque no lo hacemos rápidamente". "Sólo nos toma 10 minutos comer y después nos congelamos una hora en el patio". "Y fuera de eso siempre a la carrera, y las muchachas no hacen otra cosa que gritar, me enferman"). El resto de las críticas se refiere indirectamente al tiempo restringido (los franceses son muy cochinos, nos botan la comida a la cara", "somos 8 por mesa; se coloca una bandeja en el centro y todo el mundo se bota. . como locos").

\footnotetext{
${ }^{11}$ Estas observaciones sólo conciernen a 6 niños extranjeros y a su homólogo francés. 
En las observaciones ${ }^{12}$ pude efectivamente notar que el tiempo destinado para comer era muy breve, de 15 a 20 minutos en todas las escuelas. Como la insonorización no era suficiente, las relaciones sociales de los niños entre sí, de los niños con el personal de servicio o con los prefectos de vigilancia eran bastante agresivas y violentas - gritos, insultos, empujones, golpes-. Para los prefectos esta actividad es un control que tiene como objetivo impedir los accidentes y el desorden en demasía; no la consideran como una labor educativa tan importante como la clase; y así lo manifestaron durante las entrevistas. Se centran en la represión, castigos y amenazas, sin proporcionar modelos de conducta; por ejemplo, dar algunas normas para comer en grupo. De hecho, el personal de servicio desempeña el mismo papel, sin que nadie pueda medir el alcance educativo de su labor.

El análisis de "tiempo libre" en la escuela permitió hacer comparaciones espontáneas entre ésta y las normas del país de origen. Sucede que en esas culturas las jornadas de estudio, las horas de comida difieren de las francesas. De acuerdo con las entrevistas, fuera de los días de fiesta, dichas familias conservan el ritmo y organización de su país: la hora de acostar a los niños es la de "allá". Y como dice una española joven, "el domingo todo se hace más despacio y se almuerza después de las dos".

\section{LA EVOLUCION DEL PROCESO DE TRANSCULTURACION}

En la medida en que su estadía en Francia se prolonga, se presentan dos clases de modificaciones. En primera instancia, la representación de su cultura de origen se fija, se "folcloriza" por el hecho de encontrarse lejos de su país (Vásquez y Araujo, 1988): en otros términos conservan las normas correspondientes a la representación que los franceses se hacen de su propia cultura. Por ello se nota una creciente diferencia entre algunas normas y valores actualmente en uso en su país y aquellas que esos mismos grupos conservan en el extranjero.

Dichas comunidades terminan adoptando algunas de las normas predominantes en Francia. Al comienzo, se percibe como una limitante que debe ser obedecida en cualquier tipo de relación con la institución o con las personas que la representan. Individualmente tratan "De hacerlo a la francesa": una fórmula de cortesía, llegar puntual a la cita, dar respuesta por escrito, que se perciben como muy "francesas". Un rasgo lúdico caracteriza esta etapa ${ }^{13}$; es como si jugaran a "ser franceses"; ese proceso implica comparar y comprender que los dos sistemas son posibles y que, paralelamente se refieren a contextos e imperativos distintos.

Sin embargo, se compenetra tanto que el inmigrante o exilado sólo se percata de su gran cambio cuando regresa temporalmente a su país o cuando se dan en-encuentros casuales en Francia, con su familia o con antiguos amigos. Volvimos a encontrarnos con por lo menos un $20 \%$ de las personas con las cuales habíamos sostenido entrevistas: sus modificaciones en cuanto a norma de tiempo son flagrantes. Es como si esos niños y adolescentes, hoy adultos jóvenes, hubieran olvidado sus opiniones respecto al tiempo de los "franceses". En su discurso actual critican a sus compatriotas el mismo comportamiento que tenían y que tanto apreciaban a los seis o siete años; recurren a los mismos argumentos que antes no podían soportar: "Mi tío me pidió que lo acompañara al

\footnotetext{
${ }^{12}$ Como compartí los comedores escolares, puedo afirmar que la crítica sobre la calidad de la comida se debe a diferencias culturales. (Vásquez, 1986).

${ }^{13}$ Con frecuencia a esos "papeles" se agregan toques humorísticos. Lo interpretamos como una toma de distancia indispensable en algunos individuos. (Vásquez y Araujo, op. cit.).
} 
Louvre; ¡teníamos cita a las tres y llegó con una hora de retraso!, jincreíble! debe pensar que no tengo nada que hacer". "Una antigua compañera me llama; está de paso porParís, le digo que almorcemos a las doce en el restaurante. Y nada que hacer. Llegó casi a las dos; en ningún restaurante querían atendernos. . . .y le dije que llegara a tiempo!". "Es terrible cuando voy allá: invito al cine y todo el mundo se demora tanto, que siempre llegamos tarde. Palabra que no tienen ni idea de usar el reloj". "Paseaba en Río de Janeiro y los demás me decían por qué te apresuras tanto; no caminas, corres". Es sorprendente que esta última frase fue usada por muchos niños en las primeras entrevistas, cuando describían a los franceses por la calle. Multipliqué los ejemplos porque ilustran muy bien esa especie de amnesia que encontramos en los individuos cuando transpasan la primera etapa de transculturación (Vásquez y Araujo, op. cit.).

Sin embargo, pudimos comprobar un hecho curioso en las reuniones sociales $u$ organizadas por instituciones de la comunidad, aún en casos en los cuales los individuos han llegado a adoptar las normas francesas. Se dice que en su actividad cotidiana cada individuo se apropia de los rasgos característicos del código temporal francés: la puntualidad en las citas y la rapidez en las actividades y desplazamientos. Así pues, en reuniones sociales con compatriotas o en actividades organizadas por sus instituciones, se siguen las normas del país de origen; interrogados durante la actividad, individualmente, y sin testigos, ellos subestiman dichas normas y responsabilizan a los demás miembros de la comunidad. Una española nos confiesa durante una representación de teatro, "con esos españoles no se puede organizar nada; ¡siempre llegan tarde! ". Notemos que se refiere a los "españoles" como a los "otros"; olvida así su origen: este alejamiento de la comunidad es también muy corriente después de varios años de transculturación. En esta etapa, en cuanto a las normas del tiempo, podemos decir que los individuos administran simultáneamente los dos códigos, pero que adoptan prioritariamente las normas francesas.

\section{EL PAPEL DE LA INSTITUCION ESCOLAR}

Durante las actividades en la escuela, tanto el personal administrativo como el docente, ambos tratan de dar prioridad a los aspectos ligados al aprendizaje de las materias "escolares". Probablemente la selección se determina según criterios de promoción. Por el contrario, en muy contadas ocasiones, todo lo relativo al comportamiento se interpreta como directamente implicado en el rol pedagógico de la institución, así se trate del aprendizaje de materias o de actividades al interior de la escuela; dado el caso se le percibe como un problema de disciplina, dentro del marco de juicios de valor. Como si la escuela sólo asumiera de manera oficial unos de los aspectos de aquello que ocurre en su seno, o como si no tomara en cuenta la totalidad. Ya lo dijimos, la escuela es, después de la familia, el principal universo de socialización al cual se someten los niños de países industrializados, ocho horas diarias, durante diez años por lo menos. En el caso de niños extranjeros, como las familias no pueden asumir ese rol, es la escuela quien le propone modelos de comportamiento socialmente aceptados en Francia.

Las normas del tiempo se enmarcan dentro de la organización global de la escuela y se perciben como normas universales o como si fuesen las únicas posibles.

Por ejemplo, directores y docentes se sorprenden al saber que en otros países las entradas y salidas de clase no se hacen en fila; a menudo el comentario es "eso es desorden". Sin embargo, cabría agregar que hay diferencias de opiniones y de comportamientos entre el personal docente y el administrativo según si actúan como institución o como individuo. No se dispuso de medios técnicos para poder apreciar el 
alcance de dicha diferencia, pero en este sentido llaman la atención tres clases de hechos: según la escuela ya citada (Vásquez, 1981), se sostuvieron cortas entrevistas con los directores de las escuelas de un barrio de París y en ellas se pudieron constatar actitudes personales variables con respecto al tiempo, el propio y el de los demás; ello contrasta con una posición uniforme en cuanto a las normas y actividades exigidas. En muchas oportunidades tuve ocasión de discutir esos datos con algunos docentes; no se sienten implicados en esas observaciones; se diría que dichas exigencias se refieren al tiempo del profesor vecino. Por último, los institutores observados no piensan que actúan así, aunque se trate de ellos y aunque sus acciones se tengan en cuenta. Por ello el "tiempo de la institución" no coincide con el "tiempo de los individuos que deben representarla". Me parece que sería interesante explorar esa vía, pues podríamos preguntarnos cómo se determinan las normas del tiempo de una institución y de qué manera se imponen a sus miembros.

Efectivamente, es curioso comprobar que los individuos no se reconocen en las normas que aplican; podría plantearse la hipótesis de que la escuela está formando a los niños para una sociedad industrializada que aún no se ha implantado completamente, y sin que sus funcionarios sean conscientes de ello.

Las normas del tiempo que se aplican en la escuela no son intrínsicamente buenas ni al aprendizaje ni a su eficaz funcionamiento; las normas del restaurante escolar tampoco simbolizan las normas del "buen comer" en Francia, pero si configuran una generalización de la comida rápida. Podríamos imaginar que todo ello es un aprendizaje que pretende organizar y controlar sistemáticamente el trabajo social; un entrenamiento para adelantar tareas en las cuales se controla y se divide el tiempo, tareas en las cuales la velocidad es fundamental con respecto a los demás valores.

\section{BIBLIOGRAFIA}

Bachelard (G.) 1963. La Dialectique de la durée, Paris, PUF.

Chombart de Lauwe (M.-J.) et Ballan (C.) 1976. Enfants de l'image, Paris, Payot.

Gras (A.) 1979. Sociologie des ruptures, Paris, PUF.

Grossin (W.) 1975. "Les Cultures et le temps”, in: L’Année Sociologique, vol. 26.

Grossin (W.) 1986. "Le Temps industriel: une représentation du temps désormais contestée", in: Sociétés, 9, Juin.

Hall (E.T.) 1959. The Silent Language, New York, Doubleday.

Rezsohazy (R.) 1981. "Langages multiples sur le temps", in: Cahiers de l'Institut de Linguistique de Louvazn.

Rezsohazy (R.) 1986. "Les Mutations sociales récentes et les changements de la conception du temps", in: R.I.S.S., 107.

Samuel (N.) et Romer (M.) 1984. Le Temps libre: un temps social, Paris, Méridiens.

Soukup-Stepan (S.) et Vasquez (A.) 1985. "La Representation du temps chez les enfants et adolescents immigrés et exilés", in: Psychologie Scolaire, 54. 
Spindler (G.) 1982. "Concluding Remarks", in: Doing the Ethnography of Schooling, New York, Hole, Rinchart \& Winston.

Vasquez (A.) 1980. "Le Temps social: enfants étrangers á l'école française". in: Enfance, 3.

Vasquez (A.) 1981. "Guelques données sur la scolarite des enfants immigrés á Paris", in: Revue Internationale des Sciences de l'Education, 4.

Vasquez (A.) 1983. "Temps social Temps culturel”, in: Enfance, 1.

Vasquez (A.) et Proux (M.) 1984. "'La Maitresse dit queje suis lent': Représentation de l'école Française dans le discours d'élèves immigrés", in: International Review of Education, (UNESCO), XXX.

Vasquez (A.) 1986. "Se nourrir de nostalgie. Les conduites alimentaires des enfants étrangers en France", in: Enfance, 1.

Vasquez (A.) et Araujo (A.M.) Exils latino-américains. La malédiction d' Ulysse, Paris, L' Harmattan, 1988.

Wilcox (K.) 1982. "Methods in Ethrography", in: Spindler, Doing the Ethnography of Schooling, New York, Hole, Rinehart \& Winston. 